\title{
Lubrication State Recognition Based on Energy Characteristics of Friction Vibration with EEMD and SVM
}

\author{
Hai-jie Yu $\mathbb{D}^{D}$, Hai-jun Wei $\mathbb{C}^{D}$, Jing-ming Li $\mathbb{D}^{D}$, Da-ping Zhou, Li-dui Wei, and Hong Liu \\ Merchant Marine College, Shanghai Maritime University, Shanghai 201306, China \\ Correspondence should be addressed to Hai-jun Wei; haijun_welson@163.com
}

Received 11 March 2021; Revised 10 April 2021; Accepted 19 April 2021; Published 23 April 2021

Academic Editor: Franco Concli

Copyright ( $\odot 2021$ Hai-jie Yu et al. This is an open access article distributed under the Creative Commons Attribution License, which permits unrestricted use, distribution, and reproduction in any medium, provided the original work is properly cited.

In order to identify different lubrication states, lubrication experiments were carried out on a Bruker UMT-3 tester. The experimental results show that the frequency band energy characteristics of friction vibration signals are different under different lubrication states. Based on this, a lubrication state recognition method with ensemble empirical mode decomposition (EEMD) and support vector machine (SVM) was proposed. The vibration signals were decomposed into a finite number of stationary intrinsic mode functions (IMFs) with the EEMD method. The first six IMF components containing the main friction information were retained to calculate the energy ratio and construct the feature vector. The experimental results show that the mixed lubrication state can be identified by hundred percent, and there is a slight confusion between boundary lubrication and dry friction. The results show that frequency band energy of friction vibration signals is an effective feature to identify different lubrication states, and the proposed method can be used to identify different lubrication states.

\section{Introduction}

It is well known that friction vibration is caused by the relative movement of friction pairs. Friction vibration can reflect the characteristics and wear state of the friction system. Compared with friction coefficient, wear surface, and wear debris, friction vibration signals can be acquired online and in real-time without affecting the normal operation of the equipment. Real-time monitoring of equipment lubrication status is of great help to the efficient operation and maintenance of machinery and equipment.

As a typical nonlinear signal, the feature extraction of friction vibration has always been a difficulty in research. In recent years, the study mainly focuses on the qualitative analysis of friction vibration, but the quantitative analysis is rarely reported. Sun decomposed the vibration signals of reciprocating sliding friction pair by wavelet packet and analyzed the chaotic characteristics of the friction vibration [1]. Liu et al. analyzed the correlation between the frictional vibration in the normal and tangential directions and pointed out that the friction vibration in different directions has strong correlation [2]. Li et al. made multifractal analysis of the friction vibration signals in the running-in process [3].
In recently years, empirical mode decomposition (EMD) has been widely used to deal with nonlinear signal problems [4-7]. It adaptively decomposes signals into several stationary basic mode components by subtracting the local mean of signals in an iterative way. Because of the defects of the algorithm, false intrinsic mode functions may be generated in the decomposition results. Therefore, Huang proposed the ensemble empirical mode decomposition method (EEMD), which solves the modal aliasing problem of EMD by adding white noise components to the original signals to maintain the continuity of signals in different regions [8]. The most common method of EMD and its improved algorithm in mechanical fault diagnosis is to combine this method with other technologies to extract fault frequency [9-13]. However, there is no obvious periodicity in friction vibration and it is difficult to distinguish the lubrication state by the change of frequency. When the lubrication state changes, the energy in the same frequency band of the friction vibration signal will have a big difference. The signal energy in these frequency bands contains the main friction information, and the change of the signal energy in one or several frequency bands represents the change of the lubrication states. Therefore, the lubrication 
states can be distinguished according to the change of frequency band energy.

Support vector machine (SVM) is an intelligent optimization algorithm proposed by Vapnik et al. in 1995 on the basis of statistical learning theory $[14,15]$. Based on the principle of structural risk minimization, the SVM can solve practical problems such as small samples, high dimensions, nonlinearity, and local minimum points well. A large number of studies have shown that SVM parameters are the main factors affecting the performance of support vector machines. The differential evolution (DE) algorithm is an optimization algorithm of heuristic parallel random search based on floating-point vector coding proposed by Storn and Price in 1995 [16]. The principle of the algorithm is relatively simple with fewer control parameters. The algorithm has a strong global search ability and robustness and can improve the speed of optimization. In recent years, scholars have done a lot of work to improve the optimization performance of this method. In this paper, in order to improve the classification accuracy of support vector machine, the kernel parameters and penalty parameter of SVM are optimized by using the DE algorithm.

Based on the energy distribution characteristics of friction vibration, a lubrication state recognition method combining EEMD and SVM was proposed in this article. The vibration signals were decomposed by EEMD, and the energy ratio of the high-order intrinsic mode function (IMF) components containing the main friction information was calculated. Then, the standard mode feature vector was constructed with the energy ratio as the element, and the different lubrication states were identified by the SVM.

The innovations and main contributions of this paper are as follows:

(1) Frequency band energy is innovatively used as a characteristic to distinguish different lubrication states

(2) The IMFs are innovatively applied to construct the energy eigenvector of frequency band

(3) A lubrication state identification method based on EEMD and SVM is proposed to effectively monitor the lubrication state of equipment

The remainder of this paper is organized as follows: Section 2 presents the EEMD method, SVM method, DE algorithm, and the recognition method. The design and implementation of operational different lubrication states' tests are provided in Section 3. The results and discussion have been presented in Section 4. Finally, Section 5 would conclude the paper.

\section{Method}

2.1. EEMD. To solve the modal mixing problem of EMD, EEMD was invented on the basis of EMD algorithm in 2009 [8]. By adding white noise components to the original signal, the signals in different regions are kept continuous and the degree of mode aliasing is reduced. The decomposition steps are as follows:
(1) A Gaussian white noise is added to the original signal to produce a new signal, that is,

$$
x_{i}(t)=x(t)+n_{i}(t),
$$

where $x(t)$ is the original signal and $n_{i}(t)$ is the Gaussian white noise.

(2) The signal $x_{i}(t)$ is decomposed by EMD, that is,

$$
x_{i}(t)=\sum_{j=1}^{J} c_{i, j}(t)+r_{i, j}(t)
$$

where $c_{i, j}(t)$ is the $j^{\text {th }}$ IMF decomposed after adding white noise for the $i^{\text {th }}$ time and $r_{i, j}(t)$ is the remainder term.

(3) Repeat steps (1) and (2) for $m$ times, and add white noise signals with different amplitudes to each decomposition to get the IMF set as follows: $\left\{c_{1, j}(t)\right.$, $\left.c_{2, j}(t), \ldots, c_{m, j}(t) j=1,2, \ldots, J\right\}$.

(4) Based on the principle that the statistical mean value of unrelated sequences is zero, the average calculation is carried out for the abovementioned corresponding IMF:

$$
c_{j}(t)=\frac{1}{m} \sum_{i=1}^{m} c_{i, j}(t)
$$

where $c_{j}(t)$ is the $j^{\text {th }}$ IMF decomposed by EEMD, $i=1,2, \ldots, m$, and $j=1,2, \ldots, J$.

2.2. SVM. SVM classification is a machine learning method based on statistical learning theory and structural risk minimization. It is applicable to both linearly separable and linearly nonseparable samples. For more details, please refer to [17].

With the sample $\left(x_{i}, y_{i}\right)$ $\left(x_{i} \in R^{d} ; y_{i} \in\{-1,+1\} ; i=1,2, \ldots, n\right), x_{i}$ is a feature vector and $y_{i}$ is class label. If the sample is linearly separable, then SVM transforms the classification problem into a convex quadratic optimization problem, as shown in the following equation:

$$
\left\{\min \frac{1}{2}\|\omega\|^{2}+C \sum_{i=1}^{n} \xi_{i} \text { s.t. } y_{i}\left[\left(\omega \cdot x_{i}\right)+b\right] \geq 1-\xi_{i},\right.
$$

where $\omega$ is the weight vector, $C$ is the penalty factor, $\xi$ is the relaxation factor, and $b$ is the bias.

The dual description of the above optimization problem is obtained by Lagrange operator. Under the condition that $y_{i}\left[\left(\omega \cdot x_{i}\right)+b\right]=1$, the classification decision function can be obtained, that is,

$$
f(x)=\operatorname{sgn}\left\{\sum_{i} \alpha_{i} y_{i}\left(x_{i} \cdot x\right)+b^{*}\right\},
$$

where $\alpha_{i}$ is the Lagrange coefficient.

If the samples are linearly nonseparable, the samples in the input space can be mapped into the high-dimensional 
linearly separable feature space by nonlinear mapping, and the optimal classification decision function in the feature vector can be obtained by kernel function, that is,

$$
f(x)=\operatorname{sgn}\left\{\sum_{i} \alpha_{i}^{*} y_{i} K\left(x_{i}, x_{j}\right)+b^{*}\right\},
$$

where $K\left(x_{i}, x_{j}\right)$ is the kernel function.

2.3. Differential Evolution Algorithm. The basic idea of $\mathrm{DE}$ is to extract the search step and direction information from the current population and add random difference and crossover to improve the diversity of the population. After the above mutation and crossover operation, a temporary population is generated. Then, one-to-one selection of the two populations is carried out based on greedy thought to generate a new generation of population. The population evolves continuously according to the above method until the termination condition of the algorithm is satisfied. The details are described as follows:

(1) Four different individuals are randomly selected from the population to generate a difference vector to mutate the optimal individuals of each generation, which can not only improve the convergence speed of the algorithm but also maintain a high population diversity to a certain extent, that is,

$v_{i}^{g+1}=x_{\text {best }}^{g+1}+k\left[\left(x_{s_{1}}^{g+1}-x_{s_{2}}^{g+1}\right)+\left(x_{s_{3}}^{g+1}-x_{s_{4}}^{g+1}\right)\right]$,

where $v_{i}^{g+1}$ is the mutant individual obtained from each individual $x_{i}^{g}$ of $g$ generation by mutation operation, $x_{\text {best }}^{g+1}$ is the best individual of $g+1$ generation, and $k$ is the scaling factor.

(2) In order to improve the diversity of the population, the crossover operation mode is

$$
y_{i}^{g+1}= \begin{cases}v_{i, j}^{g+1}, & \operatorname{rand}(j) \leq \mathrm{CR}, \\ x_{i, j}^{g+1}, & \operatorname{rand}(j)>\mathrm{CR},\end{cases}
$$

where $\operatorname{rand}(j)$ is a random value on $[0,1]$ and the crossover rate (CR) is a specified constant on $[0,1]$.

(3) If the values of the parameters exceed the corresponding bounds, they will be randomly and uniformly reinitialized within the given range. Then, the target function values of all test vectors are evaluated and selected. If the objective function value of the test vector is less than or equal to the objective function value of the corresponding objective vector, the next generation replaces the objective vector with the test vector. Otherwise, the target vector will be retained for the next generation. The selection operation can be represented as follows:

$$
x_{i}^{g+1}=\left\{\begin{array}{l}
y_{i}^{g+1}, f\left(y_{i}^{g+1}\right)<f\left(x_{i}^{g}\right), \\
y_{i}^{g+1}, f\left(y_{i}^{g+1}\right)<f\left(x_{i}^{g}\right),
\end{array}\right.
$$

where $f$ is the target function.
2.4. Recognition Method. The collisions and breakage of the rough peak between the friction pairs are characterized by microimpact and random distribution on the contact interface, which stimulates the high-frequency dynamic response of the coupling system [18]. Therefore, the lower orders of IMF components should be removed and the higher orders of IMF components should be retained. As shown in Figure 1, the proposed lubrication state recognition method based on energy characteristics with EEMD and SVM is described as follows:

(1) Under the states of mixed lubrication, boundary lubrication, and dry friction, the samples are sampled several times at a certain sampling frequency to obtain enough samples.

(2) The collected vibration signals are decomposed by EEMD, and several IMF components are obtained.

(3) The energy ratio of the first $n$ IMF components is calculated:

$$
\begin{aligned}
& E_{j}=\int_{-\infty}^{+\infty} c_{j}^{2}(t) \mathrm{d} t \quad j=1,2,3, \ldots, n, \\
& T_{j}=\frac{E_{j}}{\sum_{j=1}^{n} E_{j}},
\end{aligned}
$$

where $E_{j}$ is the energy of the $j^{\text {th }}$ IMF component and $T_{j}$ is the energy proportion of the $j^{\text {th }}$ IMF component.

(4) The feature vector $T=\left[T_{1}, T_{2}, \ldots, T_{j}\right]$ is constructed.

(5) Lubrication states are classified and identified with SVM.

\section{Experiment}

3.1. Apparatus. The experiments were conducted on a piece of commercial equipment, Bruker UMT-3 tester, as illustrated in Figure 2. The equipment is able to control the disk rotational speed and the load. Friction force was measured by the sensors of the equipment. A triaxial acceleration sensor, fixed on the pin specimen (model 356B17ICP, РCB Piezotronics Company) with a range of $\pm 5 \mathrm{~g}$ and a sensitivity of $1000 \mathrm{mv} / \mathrm{g}$, was used to measure the vibration signal. A data acquisition system (VibPilot, $m+p$ international) was used to collect the data.

3.2. Experimental Method. In order to obtain the standard mode feature vectors of typical states, it is necessary to carry out experiments on the experimental bench to obtain the friction vibration signals under different lubrication states by controlling the amount of lubricating oil to change the lubrication states and according to the friction coefficient to judge the lubrication states. First, the experiment was carried out under the condition of oil lubrication. When the lubrication experiment lasted to the $50^{\text {th }} \mathrm{min}$, the severe wear experiment without lubrication was carried out, at which dry 


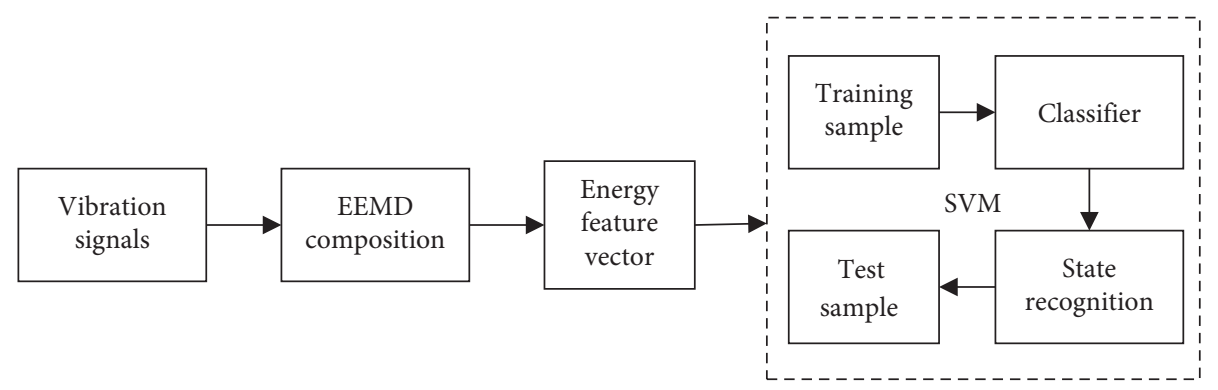

FIGURE 1: States' recognition flowchart.

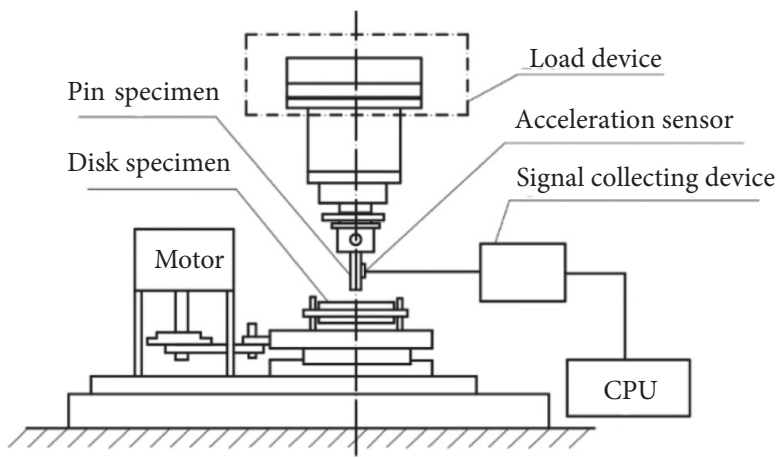

(a)

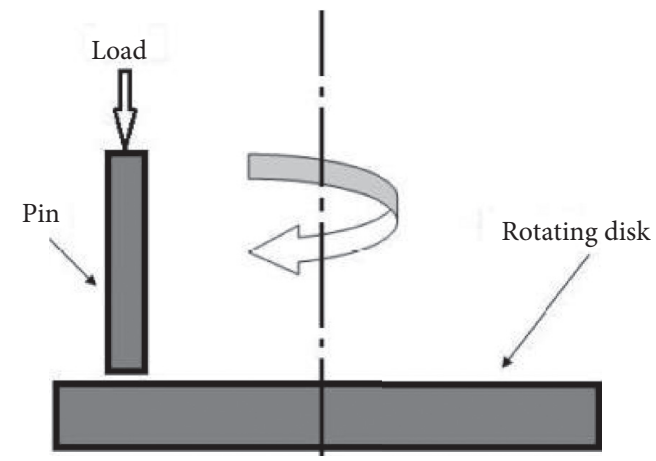

(b)

FIgUre 2: Schematic diagram of the UMT-3 tester. (a) Experiment device and (b) tribological pair.

absorbent cottons were used to clean the oil. Meanwhile, the vibration signals were collected by using the triaxial acceleration sensor with a sampling frequency of $51200 \mathrm{~Hz}$ and a sampling duration of $2 \mathrm{~s}$ per minute.

\section{Results and Discussion}

4.1. Different Lubrication States. The whole experimental process is shown in Figure 3. In the actual mechanical operation, the friction pairs are usually in the mixed lubrication state, and the friction coefficient in this state is about 0.1 . In the beginning stage, the friction coefficient experienced a short decline and then entered a stable weak fluctuation state, corresponding to the running-in wear stage and the stable wear state, respectively, which were analyzed in detailed in [19]. Until the $50^{\text {th }}$ minute, the oil was sucked away, and there was only a very small amount of adsorbed oil film between the friction pairs. The lubrication state changed from the mixed lubrication to the boundary lubrication with the friction factor varied between 0.25 and 0.4 . With the aggravation of wear, the oil films in the friction pairs were destroyed. The lubrication state changed from the boundary lubrication to the dry friction with large fluctuation of friction factor at the $70^{\text {th }}$ minute, under which the friction factor was greater than 0.4 .

Figure 4 shows the spectrum diagram of the vibration signals in different lubrication states. As analyzed in the literature [18], the friction vibration can be divided into low-frequency and medium-high-frequency parts. The low-frequency part corresponds to the eigenfrequency and coupling frequency of the system. The medium-high-frequency part is closely

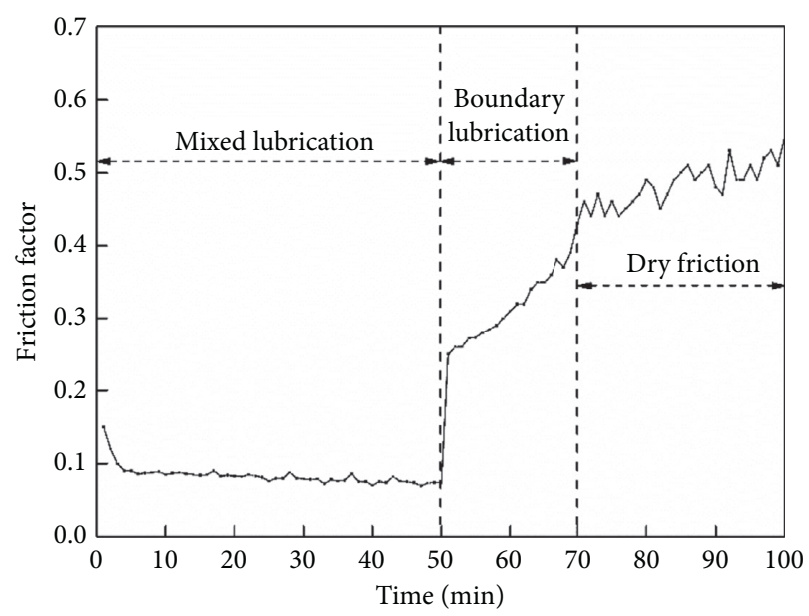

FIgURE 3: Temporal variation of friction factor in different lubrication states.

related to the surface characteristics of the friction pair. The impact and fracture of the microconvex bodies are characterized by microimpact and randomly distributed on the contact interface, which stimulates the medium-high-frequency dynamic response of the coupling system. In the process of deterioration of lubrication state, the amplitude of high-frequency components increases continuously, which is very useful to state recognition.

4.2. Energy Feature Vectors. The vibration signals collected during the experiment were decomposed by EEMD. The 


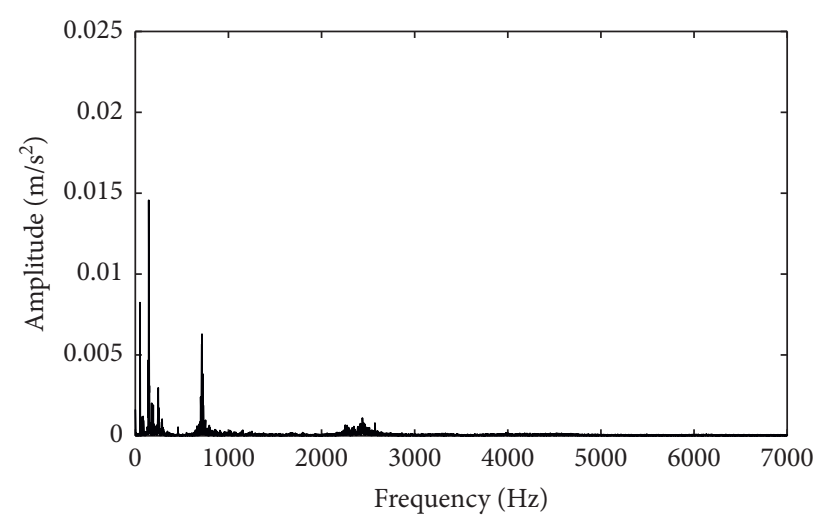

(a)

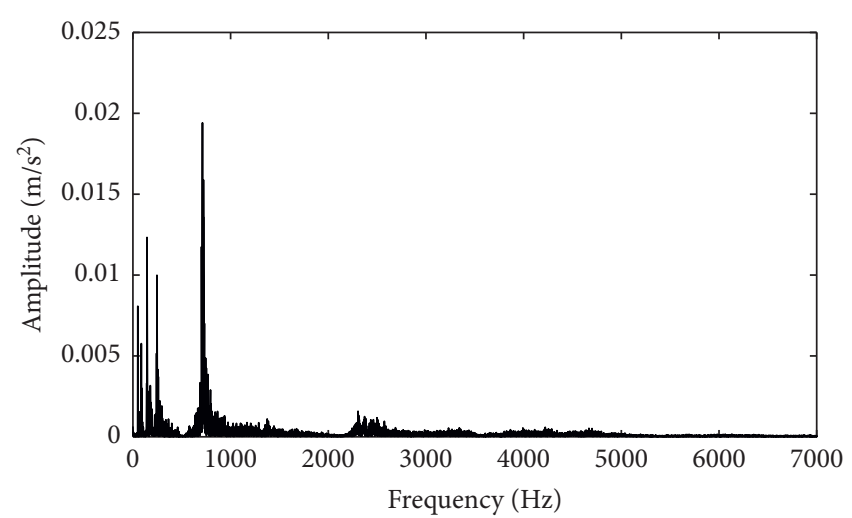

(b)

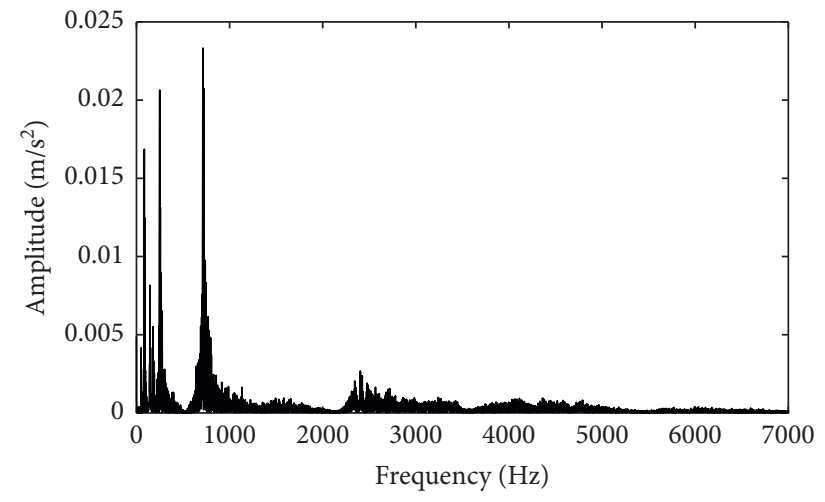

(c)

FiguRe 4: Spectrum diagram of the vibration signal in different lubrication states. (a) Mixed lubrication (20 ${ }^{\text {th }}$ min), (b) boundary lubrication $\left(60^{\text {th }} \mathrm{min}\right)$, and (c) dry friction $\left(80^{\text {th }} \mathrm{min}\right)$.

ensemble average times and the amplitude coefficient of Gaussian white noise were 100 and 0.4, respectively [3]. Figure 5 shows the EEMD decomposition results of friction vibration signals in the dry friction $\left(80^{\text {th }} \mathrm{min}\right)$. The original signal was decomposed into $10 \mathrm{IMF}$ components $c_{1}-c_{10}$ and a remainder term $r_{10}$. The IMF components represent frequency band components of the original signal and are arranged in order from high to low frequencies. The remainder term is actually a trend line, that is, the wave with very low frequency (very long period), which can be regarded as the foundation of other IMF components. The characteristics cannot be directly seen from components and remainder term.

In this paper, the first 6 order components were retained to calculate the energy ratio and construct the feature vectors. Figure 6 shows the energy ratio of the friction pair under three states. As expected, the energy distribution in different states was markedly different.
4.3. Lubrication State Classification and Recognition with $S V M$. In order to illustrate the accuracy of the method proposed in this article, the 100 samples obtained from the experiment were divided into two groups. 50 samples were taken out as training samples, and the remaining 50 samples were taken as test samples. Considering the relatively large number of training samples and the small number of features, polynomial kernel was used as the kernel function of SVM [12]. Also, the differential evolution algorithm was used to obtain the optimal penalty parameter and kernel parameters.

The results of state recognition are shown in the confusion matrix in Figure 7. In this confusion matrix, all 25 samples in the mixed lubrication state were accurately identified. However, 2 out of 10 samples in the boundary lubrication state were incorrectly recognized as dry friction. For the 15 samples in the dry friction state, 3 samples were incorrectly recognized as boundary lubrication. Confusion 

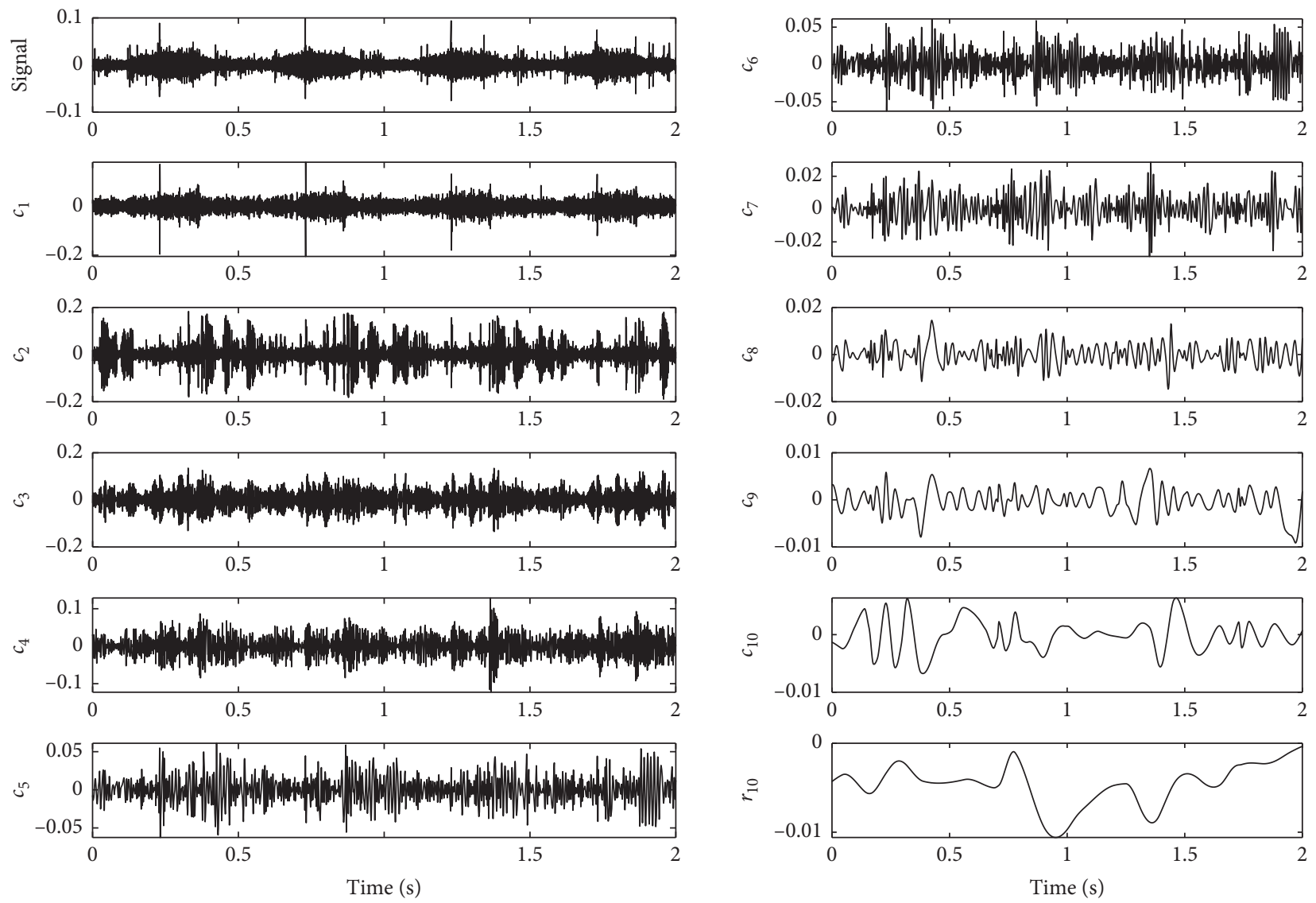

FIGURE 5: EEMD decomposition results of friction vibration signals in the dry friction.

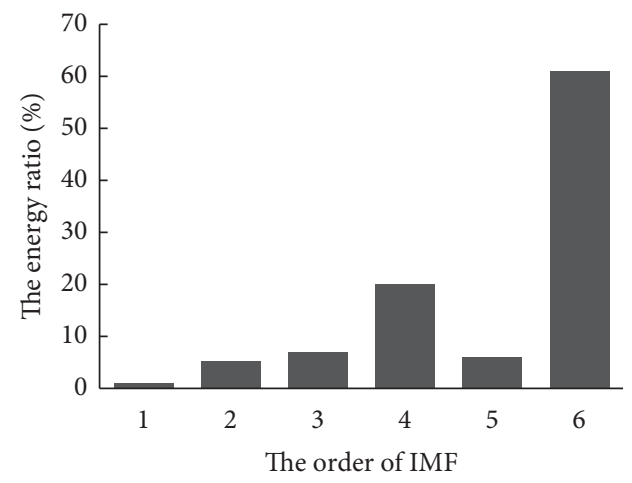

(a)

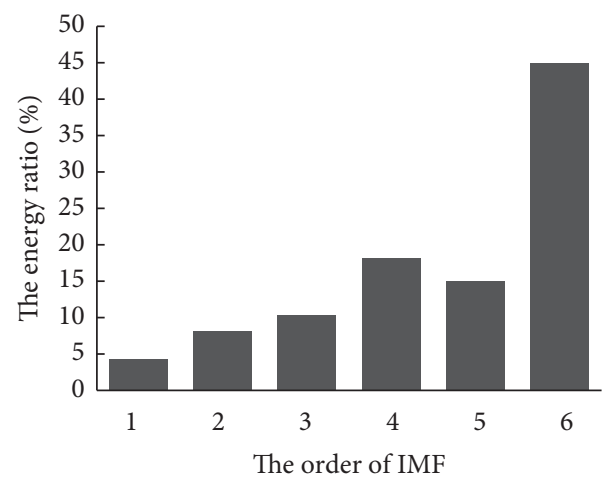

(b)

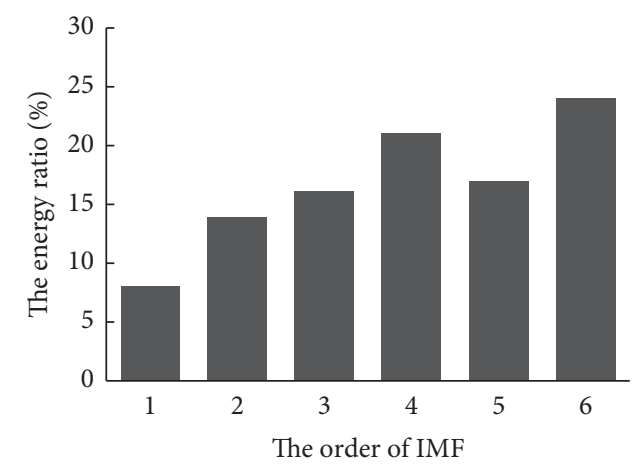

(c)

FIGURE 6: Energy ratio based on EEMD under three states. (a) Mixed lubrication $\left(20^{\text {th }} \mathrm{min}\right)$, (b) boundary lubrication $\left(60^{\text {th }} \mathrm{min}\right)$, and (c) dry friction $\left(80^{\text {th }} \mathrm{min}\right)$. 


\begin{tabular}{lccc} 
& ML & BL & DF \\
ML & 25 & 0 & 0 \\
BL & 0 & 8 & 2 \\
DF & 0 & 3 & 12 \\
\hline
\end{tabular}

FIGURE 7: Confusion matrix for the results of state recognition (ML, mixed lubrication; BL, boundary lubrication; DF, dry friction).

between the mixed lubrication state and the dry friction state can partly be explained by high degree of similarity between the two states. The results suggest that the SVM method can identify different lubrication states well.

\section{Conclusion}

In this article, vibration signals under different lubrication conditions were obtained by using the friction testing machine. The friction vibration signals were decomposed by using the EEMD method, and the medium-high-frequency components containing the main friction information were retained. The energy ratio of IMF components was calculated, and the feature vectors were constructed to provide the basis for lubrication state identification. The SVM was used to recognize the different lubrication states of friction pairs. The results illustrate that the method presented in this work can accurately recognize different lubrication states. The main conclusions are as follows:

(1) The medium-high-frequency components of the friction vibration signal contained the lubrication state information, and the retained IMF components by EEMD decomposition can be used to construct the energy feature vectors under different lubrication states.

(2) Mixed lubrication states can be accurately identified by the SVM. However, there was confusion between boundary lubrication and dry friction, and further research is needed to improve the recognition accuracy.

\section{Data Availability}

The data used to support the findings of this study are available from the corresponding author upon request.

\section{Conflicts of Interest}

The authors declare that there are no conflicts of interest regarding the publication of this paper.

\section{Acknowledgments}

This research was funded by the National High Technology Research and Development Program of China (Grant no. 2013AA040203) and the Shanghai Natural Science Foundation (Grant no. 17ZR1412700).

\section{References}

[1] D. Sun, G. Li, H. Wei, and H. Liao, "Experimental study on the chaotic attractor evolvement of the friction vibration in a running-in process," Tribology International, vol. 88, pp. 290-297, 2015.
[2] T. Liu, G. Li, H. Wei, and D. Sun, "Experimental observation of cross correlation between tangential friction vibration and normal friction vibration in a running-in process," Tribology International, vol. 97, pp. 77-88, 2016.

[3] J. Li, H. Wei, L. Fan, and L. Wei, "Multifractal detrended fluctuation analysis of frictional vibration signals in the running-in wear process," Tribology Letters, vol. 65, no. 2, 2017.

[4] K. Guo, X. Zhang, H. Li, and G. Meng, "Application of EMD method to friction signal processing," Mechanical Systems and Signal Processing, vol. 22, no. 1, pp. 248-259, 2008.

[5] Y. Lei, Z. He, and Y. Zi, "Application of the EEMD method to rotor fault diagnosis of rotating machinery," Mechanical Systems and Signal Processing, vol. 23, no. 4, pp. 1327-1338, 2009.

[6] Q. Gao, C. Duan, H. Fan, and Q. Meng, "Rotating machine fault diagnosis using empirical mode decomposition," $M e$ chanical Systems and Signal Processing, vol. 22, no. 5, pp. 1072-1081, 2008.

[7] F. Wu and L. Qu, "Diagnosis of subharmonic faults of large rotating machinery based on EMD," Mechanical Systems and Signal Processing, vol. 23, no. 2, pp. 467-475, 2009.

[8] Z. Wu and N. E. Huang, "Ensemble empirical mode decomposition: a noise-assisted data analysis method," Advances in Adaptive Data Analysis, vol. 1, no. 1, pp. 1-41, 2011.

[9] H. Wang, J. Chen, and G. Dong, "Feature extraction of rolling bearing's early weak fault based on EEMD and tunable Q-factor wavelet transform," Mechanical Systems and Signal Processing, vol. 48, no. 1-2, pp. 103-119, 2014.

[10] S. Zhao, L. Liang, G. Xu, J. Wang, and W. Zhang, "Quantitative diagnosis of a spall-like fault of a rolling element bearing by empirical mode decomposition and the approximate entropy method," Mechanical Systems and Signal Processing, vol. 40, no. 1, pp. 154-177, 2013.

[11] M. Žvokelj, S. Zupan, and I. Prebil, "Multivariate and multiscale monitoring of large-size low-speed bearings using ensemble empirical mode decomposition method combined with principal component analysis," Mechanical Systems and Signal Processing, vol. 24, no. 4, pp. 1049-1067, 2010.

[12] M. J. Zhang, K. Chai, J. Huang, and H. Chen, "Combined improved EEMD with SVM in the bearing low dimensional small sample fault diagnosis," Applied Mechanics and Materials, vol. 427-429, pp. 354-357, 2013.

[13] M. J. Zhang, J. Huang, K. Chai, and H. Chen, "Bearing binary classification intelligent diagnosis by combined improved EEMD with SVM," Applied Mechanics and Materials, vol. 341342, pp. 1066-1070, 2013.

[14] V. D. Sánchez A, "Advanced support vector machines and kernel methods," Neurocomputing, vol. 55, no. 1-2, pp. 5-20, 2003.

[15] V. N. Vapnik, The Nature of Statistical Learning Theory, Springer, Berlin, Germany, 1995.

[16] R. Storn, "Differential evolution-a simple and efficient heuristic for global optimization over continuous space," Journal of Global Optimization, vol. 11, 1997.

[17] C. Nello, An Introduction to Support Vector Machines and Other Kernel-Based Learning Methods, Cambridge University Press, Cambridge, UK, 2000.

[18] M. Di Bartolomeo, G. Lacerra, L. Baillet, E. Chatelet, and F. Massi, "Parametrical experimental and numerical analysis on friction-induced vibrations by a simple frictional system," Tribology International, vol. 112, pp. 47-57, 2017.

[19] D. Sun, G. Li, H. Wei, H. Liao, and T. Liu, "Investigation on frictional vibration behavior of tribological pairs under different wear states," Journal of Tribology, vol. 137, no. 2, Article ID 021606, 2015. 